\title{
Endoplasmic Reticulum Stress and Allergic Diseases
}

\author{
Jae Seok Jeong ${ }^{1} \cdot$ So Ri Kim ${ }^{1,2} \cdot$ Seong Ho Cho ${ }^{3}$ Yong Chul Lee ${ }^{1,2}$
}

Published online: 8 November 2017

(C) The Author(s) 2017. This article is an open access publication

\begin{abstract}
Purpose of Review In this review, we will integrate recent knowledge on endoplasmic reticulum (ER) stress and allergy, thereby highlighting the therapeutic potential of ER stress in the context of precision medicine for allergic diseases.

Recent Findings Emerging evidence suggests that allergic diseases are very heterogeneous having numerous endotypes. This leads to the new era of modern medicine, which assumes that a particular endotype-driven therapy, called precision medicine, would be more efficacious in a specific group of patients rather than in all patients. Currently, a dichotomy involving type 2/non-type 2 immune response underlies most of the studies on inflammatory and immunologic mechanisms of allergic disorders. Whereas there are several approved or investigational endotype-driven therapeutic agents targeting type 2 immune responses, investigation of mechanisms and endotype-driven interventions regarding non-type 2 immune response lags far behind.

Summary Considering that non-type 2 immune response may represent a significant proportion of allergic disease,
\end{abstract}

Jae Seok Jeong and So Ri Kim contributed equally to this work.

This article is part of the Topical Collection on Basic and Applied Science

Yong Chul Lee

leeyc@jbnu.ac.kr

1 Department of Internal Medicine, Research Center for Pulmonary Disorders, Chonbuk National University Medical School, san 2-20, Geumam-dong, Deokjin-gu, Jeonju 561-180, South Korea

2 Research Institute of Clinical Medicine of Chonbuk National University-Biomedical Research Institute of Chonbuk National University Hospital, San 2-20 Geumam-dong, Deokjin-gu, Jeonju 561-180, South Korea

3 Division of Allergy and Immunology, Internal Medicine, Morsani College of Medicine, University of South Florida, Tampa, USA particularly corticosteroid-resistant severe disease, defining a novel concept of endotype-driven approach may be essential. Recently, stress responses originate from the endoplasmic reticulum (ER) and the associated inflammatory molecular platform has been suggested as a crucial player of immune and inflammatory responses. This implies that ER stress-related pathways may represent a new endotype-driven therapeutic strategy in the treatment of allergic diseases.

Keywords Precision medicine $\cdot$ Biomarker $\cdot$ Allergic diseases $\cdot$ Endoplasmic reticulum stress $\cdot$ ER stress . Inflammation

\section{Introduction}

It is now believed that allergic diseases including bronchial asthma are very heterogeneous having numerous endotypes according to unique genetic, pharmacologic, physiologic, biologic, and immunologic mechanisms. In this context, optimal symptom control and successful treatment of allergic diseases may necessitate tailored approaches based on distinct pathophysiology in selected patients $[1,2 \bullet]$. Thus, increasing awareness of heterogeneity in allergic diseases has coincided with the emergence of endotype-driven approaches, called precision medicine $[3,4 \bullet]$. This new era of medical treatment highlights that specific group of patients may have a better response to some drugs, whereas no single drug would be efficacious for all patients. In addition, validated and qualified biomarkers are essential to identify a certain endotype better, so that researchers can define populations that will derive the most benefit from a drug [5]. For instance, early belief that bronchial asthma is the hallmark of type 2 helper $\mathrm{T}\left(\mathrm{T}_{\mathrm{H}} 2\right)$ cellmediated process led to clinical trials on evaluating therapeutic effects of anti-interleukin (IL)-5 on broad spectrum of 
severe asthmatics $[6,7]$. Initially, it failed to show significant benefits on the clinical course of the disease because inclusion of study participants was carried out in an unselected manner involving all types of asthmatics. However, later analysis revealed that anti-IL-5 can offer substantial clinical benefits in a subset of severe asthma patients with significant blood eosinophilia $[8,9]$. Therefore, to define the endotype with a stable pattern of biologic mechanism in allergic disorders will be critical for selecting any therapeutic modality more efficiently.

The endoplasmic reticulum (ER) is the largest intracellular organelle and is crucial for cellular $\mathrm{Ca}^{2+}$ storage/homeostasis and the assembly, folding, and transport of soluble/membrane proteins. Particularly as a key regulator of protein homeostasis essential for cell survival and function, ER is abundantly equipped with chaperones and enzymes that facilitate proper folding of the client proteins. Meanwhile, maintaining optimal internal conditions of ER is essential for proper folding of proteins, because ER chaperones and enzymes are highly sensitive to various stresses such as perturbations in redox state, $\mathrm{Ca}^{2+}$ concentration, and cellular energy levels [10]. Thus, altered ER homeostasis may lead to imbalance between the ER protein folding capacity and the folding load of nascent proteins, and the accumulation of misfolded/unfolded proteins in the ER lumen occurs, which is referred as ER stress. Misfolded/unfolded proteins are toxic to cells and can cause cellular stress and cell death. To maintain cellular homeostasis against this crisis, cells have evolved to efficiently detect ER stress through ER transmembrane sensors, all of which eventually trigger adaptive unfolded protein response (UPR) to restore ER functionality. Notably, a vast amount of recent study has also revealed that ER stress and UPR intersect with immunity and inflammation $[11,12]$, thereby making them as valuable therapeutic targets for treating many human disorders [13]. Particularly, emerging evidence also suggests that ER stress and UPR is closely associated with allergic inflammation through engaging with many cellular inflammatory platforms $[14 \bullet \bullet]$.

In this review, we will summarize the current concept of the pathogenesis of allergic diseases focusing on their heterogeneity in underlying immunological basis. Besides, we will highlight the therapeutic potential of ER stress and UPR in the context of precision medicine for allergic diseases through integrating the recent advances in our knowledge on this field of research.

\section{Heterogeneity in Allergic Diseases (Type 2 and Non-type 2)}

Better management of allergic diseases can be achieved through recognizing the disease heterogeneity, which is composed of diverse pathogenic mechanisms leading to clinically significant outcomes. A myriad of pathways implicated in allergic diseases have been reported to date. However, most of them may fall into type 2 or non-type 2 response based on underlying inflammatory and immunologic mechanisms. Further, several sub-endotypes may exist within each of them according to dominant cell types that orchestrate immune responses [4•].

\section{Type 2 Immune Response}

Type 2 allergic immune responses are typically characterized by eosinophilic inflammation and associated with increases of type 2 cytokines including interleukin (IL)-5, IL-13, and IL-4 in blood and affected tissues. Generally, type 2 immune responses are closely associated with atopy/allergy, in which the presence of serum antigen-specific immunoglobulin E (IgE) (mainly driven by IL-4) is the hallmark of adaptive immunity involving type 2 helper $\mathrm{T}$ cells $\left(\mathrm{T}_{\mathrm{H}} 2\right.$ cells). This immune pathway has been reported to be corticosteroid (CS)-sensitive and essential in many allergic diseases including allergic asthma, allergic rhinitis, and atopic dermatitis [4•]. However, type 2 responses may also be generated by other mechanisms irrespective of IgE reactivity to allergens and $\mathrm{T}_{\mathrm{H}} 2$ cells, as clearly demonstrated in recent researches on bronchial asthma and allergic rhinitis. In the non-allergic mechanism of type 2 cytokine production, particularly in bronchial asthma, chronic airway epithelial activation in relation to environmental factors (e.g., pollutants, irritants), viral infections, or fungal exposure induces epithelial production of IL-25, IL-33, and thymic stromal lymphopoietin (TSLP). Innate lymphoid cells (ILCs) react to these epithelium-derived cytokines, thereby producing IL-5 and IL-13 associated with airway eosinophilia and bronchial hyper-responsiveness, respectively. This mechanism may represent asthma patients who have non-atopic/ allergic and severe CS-insensitive disease while possessing eosinophilic type 2 immune responses in tissues [15]. Furthermore, the presence of this sub-endotype of type $2 \mathrm{im}$ mune responses partly explains why targeting type 2 cytokine pathway is effective in a subset of non-atopic asthmatic patients with high levels of eosinophils in blood and frequent exacerbation despite maximal current treatments including inhaled CS and/or systemic CS [16, 17]. Based on these findings, currently, there are several approved or investigational endotype-driven therapeutic agents targeting type 2 immune responses in bronchial asthma and other allergic diseases $[18$, 19]. In addition, diverse innate and adaptive immune pathways related to $T_{H} 1 / T_{H} 17$ cells, environmental exposure (e.g. smoking, occupational exposure), viruses/bacteria, and tissue injury may further modulate type 2 immune responses, particularly in less allergic form, leading to clinically more severe allergic disease with mixed eosinophilic/neutrophilic inflammation in some individuals $[20,21 \cdot 22,23]$. These findings also highlight the complex nature of the CSresistant severe allergic inflammatory process. 


\section{Non-type 2 Immune Response}

Currently, the overall proportion of asthma associated with type 2 immune responses is estimated to be approximately $50 \%$ of patients $[24,25]$. Thus, individuals with non-type 2 immune responses represent a large proportion of asthmatics. Likewise, results from several previous clinical trials demonstrating the ineffectiveness of type 2 cytokine-targeted therapies in non-phenotyped, overall groups of asthmatics may imply the presence of bronchial asthma having non-type 2 immune response [26]. However, little is understood regarding the mechanisms underlying this type of immune response and most of the knowledge is derived from studies of bronchial asthma and rhinitis. Generally, both type $17\left(\mathrm{~T}_{\mathrm{H}} 17\right)$ and type $1\left(\mathrm{~T}_{\mathrm{H}} 1\right)$ responses are often associated with neutrophilic inflammation of airways. While lung neutrophilia can be interpreted as secondary finding of CS use [27], the presence of neutrophilic inflammation has been associated with more severe clinical manifestations of bronchial asthma, more CSrefractory disease, and lower lung function in affected patients $[21 \bullet, 28 \bullet]$. In a preclinical model of asthma, adoptive transfer of allergen-specific $T_{H} 17$ cells to mice induced a chemokine (CXCL8, also known as IL-8)-mediated neutrophil influx into the lung, which was not attenuated by CS [29]. Similarly, sputum ILI7A and IL8 mRNA levels were correlated with each other and with sputum neutrophil counts in asthmatics and levels of these transcripts increased with increasing severity of asthma [30]. However, single targeted therapy blocking IL-17 receptor signaling has shown a minimal effect in subjects with inadequately controlled moderate to severe asthma in a clinical trial [31]. These results may be in line with the hypothesis that CS-insensitive severe asthma possesses mixed type 17/type 1 immune response in the background of variable type 2 immunity [21•,32]. In the same vein, the existence of a unique molecular phenotype of asthma characterized by simultaneous activation of type 17 and type 1 immune response with airway neutrophilia has been demonstrated in clustering analysis of sputum cell transcriptomics from moderate to severe asthmatic subjects [33•]. In fact, early reports showed that interferon (IFN)- $\gamma$ producing $\mathrm{T}$ cells were increased in airways of asthmatics [34] and serum concentration of IFN- $\gamma$ was elevated especially in patients with acute severe asthma [35]. More recently, IFN- $\gamma$ has been implicated in bronchial asthma pathogenesis through $\mathrm{T}_{\mathrm{H}} 2$-independent IFN- $\gamma /$ mast cell axis [36] as well as its classical effects on $\mathrm{T}_{\mathrm{H}} 2$ cells [37, 38]. However, little is known about the therapeutic effect of IFN- $\gamma$ blockade in the treatment of bronchial asthma and other allergic diseases so far. Furthermore, considering the existence of another largely unknown non-type 2 paucigranulocytic asthma (the absence of detectable inflammatory process) $\left[22,33^{\circ}\right]$, development of effective endotype-driven therapy may be further hampered by our limited knowledge on the mechanisms contributing to the non-type 2 immune response in allergic diseases. Currently, there is no approved endotypedriven therapeutic agent, targeting non-type 2 allergy [18].

\section{ER Stress and the UPR Pathways}

Three ER transmembrane sensors, including inositolrequiring enzyme $1 \alpha$ (IRE1 $\alpha$ ), double-stranded RNA-dependent protein kinase (PKR)-like ER kinase (PERK), and activating transcription factor 6 (ATF6), monitor protein homeostasis of ER lumen and transmit their information to the cytosolic compartment of cells through UPR pathways. This process can be both normal physiology and pathological phenomenon because even in normal physiological processes, such as increasing demands of protein secretion in secretory cells (e.g., plasma cells producing a large amount of immunoglobulins), cells can experience ER stress. Therefore, the canonical understanding is that UPR fine-tunes the secretory pathway of ER and attempts to reduce ER stress through reducing demand of protein folding, promoting ER-associated degradation of proteins by the ubiquitin-proteasome system (namely ERassociated degradation, ERAD), and increasing ER chaperones and enzymes helping protein folding to defend cells from ER stress. If cells fail to resolve ER stress, these adaptive responses will initiate apoptosis. Recently, in addition to these canonical UPR activities, non-canonical UPR activities are involved in connecting protein homeostasis-related cellular apparatus to a wide array of cellular events including immunity and inflammation through various mechanisms, as substantially reviewed elsewhere $[11,12]$.

IRE $1 \alpha$ is the most evolutionarily conserved sensor pathway among three UPR pathways and possesses both protein kinase activity and site-specific endoribonuclease (RNase) activity. In the presence of ER stress, IRE1 $\alpha$ is activated when an abundant ER chaperone glucose-regulated protein 78 (GRP78) dissociates from IRE1 $\alpha$. Similar mechanisms (ER stress-driven dissociation of GRP78) also explain the activation of PERK and ATF6. Direct activation of IRE1 $\alpha$ following engagement with misfolded proteins has been also demonstrated. Dissociated GRP78 preferentially binds to unfolded/misfolded proteins allowing IRE $1 \alpha$ to dimerize and autophosphorylate through its kinase activity. This leads to the activation of specific RNase activity of IRE1 $\alpha$, leading to the splicing of mRNA encoding X-box-binding protein 1 (XBP1u) and generating a spliced variant (XBP1s). XBP1s functions as a transcription factor for genes associated with lipid metabolism, immune and inflammatory responses, and cellular differentiation as well as genes traditionally associated with structural and functional expansion of ER and ERAD [39] (Fig. 1). Furthermore, through its non-specific RNase activity, IRE $1 \alpha$ has known to degrade ER-membraneassociated mRNA to reduce protein production, also known as regulated IRE1 $\alpha$-dependent decay [40]. 


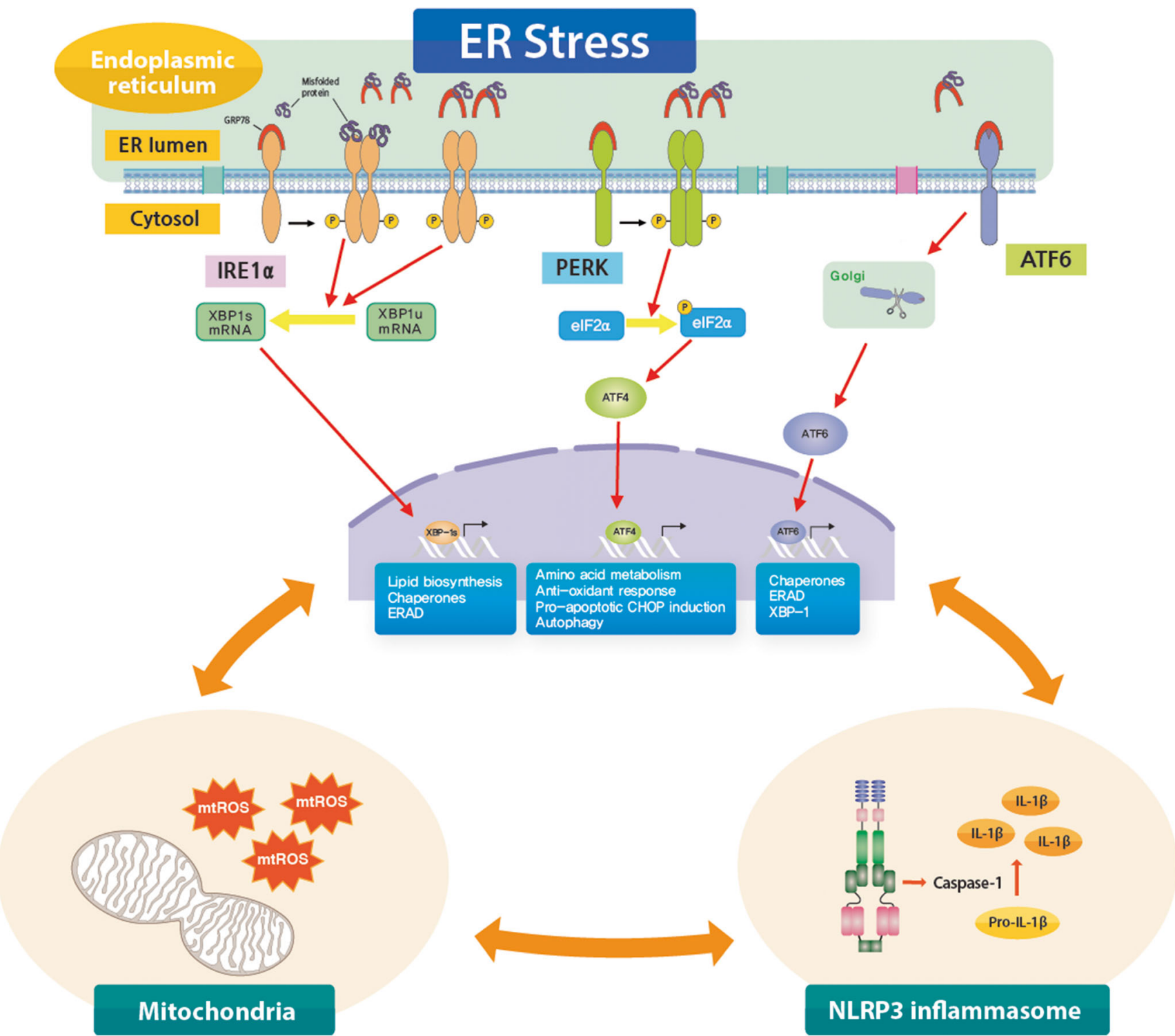

Fig. 1 Interconnection between endoplasmic reticulum (ER) stress/ unfolded protein response (UPR) pathways and mitochondria-NLRP3 inflammasome in allergic diseases. The accumulation of misfolded/ unfolded proteins in the ER lumen activates UPR, which is mediated by three ER transmembrane stress sensors including inositol-requiring $1 \alpha$ (IRE1 $\alpha$ ), double-stranded RNA-dependent protein kinase (PKR)-like ER kinase (PERK), and activating transcription factor 6 (ATF6). In a condition of ER stress, an abundant ER chaperone, glucose-regulated protein 78 (GRP78), preferentially associates with accumulated misfolded/unfolded proteins. Dissociation of GRP78 from the ER stress sensors, or direct engagement of misfolded/unfolded protein to IRE1 $\alpha$, transmits signals about folding status of ER to the cytosol and nucleus. The canonical aspect of UPR regulates the secretory pathway of ER and

Activation of PERK, regulated similarly to IRE $1 \alpha$, leads to the recruitment and phosphorylation of a translation initiation factor, eukaryotic translation initiation factor $2 \alpha$ (eIF2 $\alpha)$, through its kinase activity. Phosphorylation of eIF $2 \alpha$ inhibits synthesis of proteins through interfering the assembly of the ribosome in eukaryotic cells, thereby reducing protein folding load in ER-stressed cells. In addition, phosphorylated eIF2 $\alpha$ attempts to reduce ER stress through reducing demand of protein folding, promoting ER-associated degradation (ERAD) and gene expression involved in cell survival (e.g., autophagy), and increasing ER chaperones to defend cells from ER stress. If cells fail to resolve ER stress, these adaptive responses will initiate apoptosis, mainly through $\mathrm{C} / \mathrm{EBP}$ homologous protein (CHOP). In addition to this canonical aspect of UPR pathways, close interrelationship between ER/UPR pathways and cellular inflammatory platforms including mitochondria (e.g., oxidative stress from mitochondrial reactive oxygen species (ROS); mtROS) and NLRP3 inflammasome (an interleukin-1 $\beta$ producing platform) may be critically implicated in a unique form of corticosteroid-resistant type 2 allergic immune response

selectively induces translation of ATF4 mRNA that contains regulatory sequences such as an inhibitory upstream open reading frame. As a transcription factor, ATF4 controls amino acid metabolism, anti-oxidant response, and autophagy (an adaptive self-eating process by which cellular components are encapsulated in autophagosomes and degraded). Furthermore, PERK-mediated apoptosis occurs later during 
ER stress when causative stimuli of ER stress is strong and the other arms of UPR fail to restore protein homeostasis. This process is known to be mediated by $\mathrm{C} / \mathrm{EBP}$ homologous protein $(\mathrm{CHOP})$, which is one of the representative ER stress markers in addition to GRP78 [41•] (Fig. 1).

Lastly, on release from GRP87, ATF6 moves to the Golgi apparatus where it is cleaved to produce the functional cytosolic fragment of ATF6. Then, fragmented ATF6 is translocated to the nucleus and activates expression of chaperones and ERAD-associated proteins (Fig. 1). Many aspects of outcomes from ATF6 activation resemble those of IRE1 $\alpha /$ XBP1 pathway on the ER protein quality control system. Moreover, complete activation of the IRE $1 \alpha$ pathway is partly controlled by ATF6 pathway because further induction of XBP1 mRNA depends on ATF6 [42]. Thus, the ATF6 pathway seems to fine-tune UPR along with IRE1 $\alpha$ and PERK pathways.

\section{ER Stress as an Endotype of Severe Allergic Inflammation}

\section{Involvement of ER Stress and UPR in Various Facets of Allergic Response}

The allergic response begins in the interface between external environment and epithelium and subsequently involves diverse cell types at all levels of innate and adaptive immunity. During these processes, cells produce large amounts of secretory proteins to defend themselves against endogenous and exogenous threats and/or to efficiently communicate with other cell types for generating an organized immune response. Thus, proper functioning of ER and maintenance of protein homeostasis is critical in these cells. Although the presence of ER stress is not always interpreted as a pathological phenomenon [43], sustained and overwhelming ER stress in various cells can profoundly impact normal cellular physiology. Importantly, the influence of ER stress is just restricted to protein folding, but can intersect at many levels with immunity, thereby leading to complex chronic inflammatory disease such as allergy.

\section{Epithelial Cell-Dendritic Cell (DC) Interaction}

Before activation of various $\mathrm{T}$ cells implicated in various endotypes in allergic diseases, antigen-presenting DCs must recognize allergen and present it to T cells in draining lymph nodes. Epithelial cells are known to be a key modulator in controlling DC activation through releasing epithelialderived cytokines (e.g., IL-25, IL-33, TSLP) and endogenous-associated molecules (e.g., uric acid, ATP, HMGB1). Given the role of epithelial cells as the first line of defense, coexistence of diverse environmental insults (e.g., pollution, smoking, occupational exposure, viruses/bacteria, and simultaneous exposure to other allergens) may also converge on epithelial-DC interaction [15, 44], further shaping and characterizing the underlying endotype of allergic response. In this regard, the role of protein-secreting apparatus of epithelium is indispensable. For example, inflamed airway epithelial cells demonstrate overt signs of ER stress [45] and bronchial epithelial XBP-1 has been reported to mediate inflammation-induced $\mathrm{ER} / \mathrm{Ca}^{2+}$ store expansion which amplifies $\mathrm{Ca}^{2+}$-dependent secretion of cytokines [46]. Moreover, the IRE-1/XBP-1 arm of UPR has been proposed to be important in maintaining the integrity of airway [47] and intestinal epithelium [48]. Very recently, airway epithelial ORM (yeast)-like protein isoform 3 (ORMDL3), an ER transmembrane protein associated with ORMLD3 gene locus (17q21) well-known as a highly significant risk factor for the development of asthma [49, 50], has been demonstrated to be critically implicated in fungal allergic airway disease through ATF6-mediated activation of XBP-1 and ERAD pathway [51••]. Meanwhile, IRE1 $\alpha-\mathrm{XBP} 1$ is also a prerequisite for the proper antigen-presenting function of DC as well as DC development and survival in physiologic context [11, 52, 53]. Taken together, ER stress and UPR pathway may be a central player in the regulation of epithelial-DC interaction that is crucial for the initiation and amplification of allergic response.

\section{B Cells, Plasma Cells, and T Cells}

Activation of adaptive immunity involving various T cells and $\mathrm{B}$ cells (including secretory plasma cells which secrete high levels of antibody), which contribute to diverse endotypes of allergic response, follows the initial recognition and amplification phase that involves epithelial-DC interaction. In the draining lymph nodes, $\mathrm{T}_{\mathrm{H}} 2$ and $\mathrm{T}_{\mathrm{H}} 17$ polarization may occur

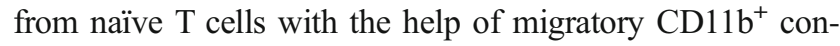
ventional DCs. Subsequently IL-4 production from $\mathrm{T}_{\mathrm{H}} 2$ cells induces class switching in B cells, and synthesis of $\mathrm{IgE}$ from plasma cells which contributes to allergen-specific sensitization for type 2 immune response. Once sensitized, repeated exposure to the same offending allergen may lead to the robust re-stimulation of these effector cells that is mainly mediated by poorly migratory CD11 $c^{\text {hi }}$ monocytic DCs [15]. In this process, XBP1 is known to be pivotal in terminal differentiation of B cells into highly secretory plasma cells through mediating expansion of the ER and synthesis of proteins required for antibody production and secretion [54]. Moreover, the IRE1 $\alpha$-XBP1 arm of UPR is thought to be important in the early developmental stage of B cells [55] and terminal differentiation of effector $\mathrm{CD}^{+} \mathrm{T}$ cells [56]. Notably, considering that XBP-1 mRNA in naïve B cells is uniquely induced by IL4 [57], this arm of UPR may be particularly important in type 2 endotype of allergic response. 


\section{Granulocytes and Macrophages}

Eosinophils and neutrophils are major effector cells in type 2 or non-type 2 immune response, respectively. However, neutrophilic inflammation may coexist with variable degrees of eosinophilic inflammation, particularly in a more severe form of the allergic disease with mixed eosinophilic/neutrophilic inflammation [20]. Alternatively activated macrophages may further fine-tune these processes through upregulation of chitinase-like proteins, one of the most abundant proteins under type 2 immune response that are involved in impaired antiviral immunity [58] and neutrophil-rich type of allergic inflammation under type 2 immunity [59]. Among diverse granulocytes, differentiation of eosinophils from progenitors of myeloid cells is known to uniquely rely on the IRE1 $\alpha$ XBP1 pathway and deletion of XBP1 results in massive defects in eosinophil maturation [60••]. Additionally, macrophages are known to associate cell surface innate toll-like receptor (TLR) signaling with intracellular IRE1 $\alpha$-XBP1 pathway-mediated secretion of pro-inflammatory cytokines (e.g., IL-6 and tumor necrosis factor) [61], thereby linking ER and UPR pathway to innate effector function in macrophages. Furthermore, at the same time, TLR signaling suppresses the ATF4-CHOP-mediated cellular apoptotic pathway to effectively coordinate innate function of macrophages [62]. These data emphasize the critical involvement of ER stress and UPR pathway in terminal effector phases of immune response as well.

\section{ER Stress and UPR in Type 2 Allergic Response}

It has been demonstrated that ER stress and UPR pathways are critically implicated in human allergic diseases and considerable data were derived from studies on allergic inflammation in the lung. In particular, increased levels of ER stress markers (e.g., GRP78, CHOP) were observed in peripheral blood mononuclear cells as well as bronchoalveolar lavage fluids from patients with bronchial asthma compared to those in healthy subjects [63••]. Furthermore, airway epithelial activation of UPR and the related increase of ER-localized chaperones were observed using in vitro HDM-exposed primary human nasal epithelial cells and bronchial epithelial cells [64] and human lung biopsy specimens from asthmatic patients [65]. Interestingly, alleviation of ER stress using chemical chaperones which promote proper folding of client proteins $[63,66]$ or ablation of UPR and related pathways [64, 65] significantly attenuates allergen-induced typical eosinophilic type 2 immune responses in animal models, thereby highlighting the role of ER stress in inducing and maintaining cardinal features of type 2 immune response.

ER stress and UPR activation can be interconnected with type 2 immune responses through various mechanisms. In an immunological context, ER stress and UPR may lead to transcriptional changes in various cell types essential for the production of certain cytokines/chemokines, thus modulating subsequent immune cell behaviors and inflammatory profiles of tissues [14••]. Moreover, increases of ER-localized chaperones that accompany ER stress is associated with transcriptional activation of UPR that may further potentiate the action of pro-inflammatory, apoptotic, and fibrotic mediators apart from immunologic mechanisms [65]. Importantly, in addition to these classically well-known inflammatory mechanisms associated with ER stress and UPR as substantially reviewed elsewhere [67], a recent study from our group highlights the connection between ER and cellular inflammatory platforms including mitochondria and NLRP3 inflammasome in a unique form of CS-resistant type 2 immune response associated with fungal sensitization [68・•0].

Respiratory fungal exposure has been regarded as a precipitating factor for severe asthma. A number of epidemiologic studies have shown that fungal sensitization is found more frequently in asthmatics severe asthma [69•, 70, 71]. In one study, over $50 \%$ of patients with severe asthma were sensitized to one or more fungi [72]. Fungal sensitization in asthma is often characterized by marked type 2 immune response associated with blood and tissue eosinophilia [2•] and may be associated with airway destruction in the later course of the disease as seen in allergic bronchopulmonary aspergillosis (ABPA) [71]. Based on this knowledge, many researchers have focused on identifying mechanisms whereby fungi can be associated with severe asthma. In our study, GRP78 is remarkably increased in lung tissues from ABPA patients compared to that in healthy subjects. In line with this result, respiratory exposure of mice to fungal allergens from Aspergillus fumigatus leads to significant increases in ER stress markers (GRP78 and CHOP) and UPR pathway proteins (phosphorylated (p) IRE1 $\alpha$-XBP1 and p-eIF2 $\alpha$-ATF4). These observations were further verified by in vitro experiments using A. fumigatus-exposed primary cultured murine tracheal epithelial cells. Notably, A. fumigatus-induced pulmonary type 2 immune responses, including eosinophilic airway inflammation and increases in the levels of serum total $/ A$. fumigatus-specific IgE and pulmonary type 2 cytokines (e.g., IL-4, IL-5, and IL-13), are remarkably improved by treatment with a potent ER stress inhibitor. In contrast, dexamethasone fails to improve A. fumigatus-induced pulmonary type $2 \mathrm{im}$ mune responses, implying that ER stress and UPR activation may be implicated in fungus-induced CS-resistant allergic inflammation. Furthermore, our results also demonstrate that oxidative stress, particularly from mitochondria (mitochondrial reactive oxygen species; mtROS) plays a key role in fungal type 2 immune response, and that a potent mtROS scavenger dramatically ameliorates $A$. fumigatus-induced CS-resistant type 2 response as well as ER stress [68]. In our unpublished data, respiratory exposure to A. fumigatus allergens also results in the activation of a cytoplasmic pattern recognition 
receptor, NLPR3, and subsequent formation of proteolytic multiprotein complex termed inflammasome, an IL-1 $\beta$ producing platform, especially in airway epithelium. Importantly, treatment with anti-IL-1 $\beta$ antibody and/or blockade of activation/assembly of NLRP3 inflammasome using NLRP3-specific inhibitor remarkably ameliorates fungiinduced CS-resistant type 2 immune responses. Considering that ER stress can cause the release of various damageassociated molecular patterns from mitochondria (e.g., mtROS, mitochondrial DNA, ATP, and calcium), which are also potent activators of cytosolic NLRP3 inflammasome [73], interconnection between ER stress, mitochondria, and the NLRP3 inflammasome may play a pivotal role in the pathogenesis of CS-resistant severe type 2 immune response (Fig. 1). In addition, nuclear translocation of NF- $\mathrm{kB}$ p 65 is remarkably increased in lung tissues from the murine model of $A$. fumigatus-induced fungal allergic lung inflammation and an ER stress regulator reduces the A. fumigatus-induced increase of NF- $\mathrm{kB}$ p65 nuclear translocation [68・•]. Moreover, treatment of mice with an NF-KB inhibitor reduces the $A$. fumigatus-induced type 2 cytokine production and eosinophilic allergic inflammation [68••]. These findings suggest the crucial implication of ER stress-associated NF-KB signaling in fungi-induced CS-resistant type 2 inflammation.

\section{ER Stress and UPR in Non-type 2 Allergic Response}

In lipopolysaccharide (LPS)-induced acute lung inflammatory animal model, ER stress has been reported to be linked to several transcriptional factors including NF- $\mathrm{kB}$ and hypoxiainducible factor $1 \alpha$, all of which play a central role in acute neutrophil-dominant inflammation and plasma exudation in the lung [41•]. In addition, LPS-induced ER stress leads to the increased expression of IL-17 in airway epithelium, thereby further potentiating ER stress and NF- $\mathrm{KB}$ activation via forming a positive feedback loop in airway epithelial cells [74•]. These data suggest that ER stress and UPR pathways may play a role in non-type 2 neutrophilic allergic response. However, the contribution of ER stress and UPR in non-type 2 immune response has been less defined compared to that in type 2 response. Notably, we previously showed that ER stress is critically implicated in the pathogenesis of bronchial asthma, particularly non-type 2 form of the disease, by using ovalbumin (OVA)/LPS-sensitized and OVA/LPS-challenged $\left(\mathrm{OVA}_{\mathrm{LPS}}-\mathrm{OVA}\right)$ mice [63••]. The classical OVA-sensitized/ challenged (OVA-OVA) mice show CS-responsive pulmonary eosinophilic type 2 inflammation. In contrast, the $\mathrm{OVA}_{\mathrm{LPS}}-\mathrm{OVA}$ mice display neutrophilic airway inflammation with mixed type 17/type 1/type 2 profiles (i.e., concurrent increases of IL-17/IFN- $\gamma$ and type 2 cytokines such as IL-4, IL-5, and IL-13 in the lung), all of which are not improved by systemic CS. Interestingly, a potent ER stress regulator significantly reduces the OVA/LPS-induced CS-resistant

\section{ER Stress}

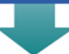

Inflammatory pathways
Epithelium

Dendritic cells

T cells

B cells and plasma cells

Granulocytes and macrophages

Nuclear factor (NF)-KB

TNF, IL-6

NLRP3 inflammasome activation

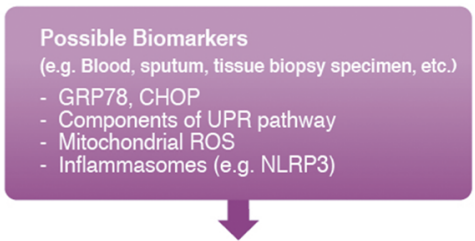

ER stress-Related Inflammatory \& Immune Response

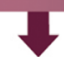

Tailored Therapy

Fig. 2 A proposed endotype-driven approach based on endoplasmic reticulum (ER) stress in allergic diseases. ER stress and unfolded protein response (UPR) pathways are closely associated with allergic immune responses involving various important cell types (e.g., epithelial cells, dendritic cells, T and B cells, granulocytes, and macrophages) and inflammatory pathways (e.g., ER stress-associated nuclear factor (NF)-KB signaling, UPR-dependent secretion of interleukin (IL)-6 and tumor necrosis factor (TNF), and NLRP3 inflammasome-mediated IL- $1 \beta$ production). Through analyzing ER stress-associated molecular profiles including ER stress markers (e.g., GRP78 and CHOP), UPR pathway components, and related inflammatory platforms (e.g., mitochondrial reactive oxygen species (ROS) and NLRP3 inflammasome) in blood, sputum, or tissue biopsy specimen from allergic patients, we may design a novel endotype-based approach in association with ER stress

neutrophilic inflammation as well as ER stress. Meanwhile, increased levels of ER stress and UPR protein are observed in lung tissues of OVA-OVA mice and administration of systemic CS markedly reduces the OVA-induced elevations of these proteins. These data imply the crucial involvement of ER stress and UPR in non-type 2 CS-resistant allergic immune responses. Further studies are needed to delineate their roles in non-type 2 immune responses.

\section{Conclusions and Perspectives}

As the beginning of a new era, precision medicine will offer a chance to effectively control and even cure allergic disease for a specific group of patients. Particularly for allergic diseases, current clinical/experimental approaches are largely based on the classification as type 2 and nontype 2 immune responses, and this seems to be quite useful, at least for type 2 response. However, investigation of 
mechanisms and endotype-driven interventions regarding non-type 2 immune response lags much behind those of type 2 responses. As described earlier, studies have shown that ER stress and UPR pathways may be involved in both types of immune responses via engagement with various important cell types and inflammatory pathways. By analyzing ER stress-associated molecular profiles including ER stress markers (e.g., GRP78 and CHOP), UPR components, and inflammatory platforms (e.g., mitochondrial ROS and NLRP3 inflammasome) in blood, sputum, or tissue biopsy specimen from allergic patients, we may verify the presence and intensity of ER stress and design a novel therapeutic strategy targeting ER stress and specific UPR pathways. We can also monitor the treatment response in these patients through selected biomarkers from ER stress-related pathways. This concept parallels a novel endotype-based approach in allergic disease in association with ER stress (Fig. 2). As CS-resistant allergic inflammation accounts for a significant proportion of the healthcare expenditure for allergic disease, understanding mechanisms behind the close involvement of ER stress in improved knowledge on this issue will permit a great chance to cure intractable allergic disease. Large-scale clinical trials and large cohort studies may be warranted to further delineate the role of ER stress in many human allergic diseases in the future.

Funding Information This study was supported by the Korea Healthcare Technology R\&D Project, Ministry of Health and Welfare, Republic of Korea, grant A121931; the Basic Science Research Program through the National Research Foundation of Korea funded by the Ministry of Science, Information and Communication Technology and Future Planning grant NRF-2014R1A2A1A01002823; and the fund of Biomedical Research Institute, Chonbuk National University Hospital.

\section{Compliance with Ethical Standards}

Conflict of Interest Dr. Lee reports grants from The Korea Healthcare Technology R\&D Project, Ministry of Health and Welfare, Republic of Korea, grants from The Basic Science Research Program through the National Research Foundation of Korea funded by the Ministry of Science, Information and Communication Technology and Future Planning, grants from Biomedical Research Institute, Chonbuk National University Hospital, during the conduct of the study. The other authors declare no conflicts of interest relevant to this manuscript.

Human and Animal Rights and Informed Consent This article does not contain any studies with human or animal subjects performed by any of the authors.

Open Access This article is distributed under the terms of the Creative Commons Attribution 4.0 International License (http:// creativecommons.org/licenses/by/4.0/), which permits unrestricted use, distribution, and reproduction in any medium, provided you give appropriate credit to the original author(s) and the source, provide a link to the Creative Commons license, and indicate if changes were made.

\section{References}

Papers of particular interest, published recently, have been highlighted as:

- Of importance

•- Of major importance

1. Anderson GP. Endotyping asthma: new insights into key pathogenic mechanisms in a complex, heterogeneous disease. Lancet. 2008;372(9643):1107-19. https://doi.org/10.1016/s0140-6736(08) 61452-x.

2. Lotvall J, Akdis CA, Bacharier LB, Bjermer L, Casale TB, Custovic A, et al. Asthma endotypes: a new approach to classification of disease entities within the asthma syndrome. J Allergy Clin Immunol. 2011;127(2):355-60. https://doi.org/10.1016/j.jaci. 2010.11.037. The practical allergy (PRACTALL) consensus report produced by experts from the European Academy of Allergy and Clinical Immunology and the American Academy of Allergy, Asthma \& Immunology. The criteria for defining asthma endotypes based on phenotypes and pathophysiology are proposed. Moreover, this report suggests a number of important examples of asthma endotypes.

3. Collins FS, Varmus H. A new initiative on precision medicine. $\mathrm{N}$ Engl J Med. 2015;372(9):793-5. https://doi.org/10.1056/ NEJMp1500523.

4. Muraro A, Lemanske RF Jr, Hellings PW, Akdis CA, Bieber T, Casale TB, et al. Precision medicine in patients with allergic diseases: airway diseases and atopic dermatitis-PRACTALL document of the European academy of allergy and clinical immunology and the American Academy of Allergy, Asthma \& Immunology. J Allergy Clin Immunol. 2016;137(5):1347-58. https://doi.org/10. 1016/j.jaci.2016.03.010. In the new era of precision medicine, this consensus document highlights the recent advances in our knowledge of the pathogenesis (endotypes) of several allergic diseases together with proposed biomarkers, thereby supporting the potential for the allergy/immunology to use the precision medicine approach.

5. Biomarkers and surrogate endpoints: preferred definitions and conceptual framework. Clin Pharmacol Ther. 2001;69(3):89-95. https://doi.org/10.1067/mcp.2001.113989.

6. Leckie MJ, ten Brinke A, Khan J, Diamant Z, O'Connor BJ, Walls $\mathrm{CM}$, et al. Effects of an interleukin-5 blocking monoclonal antibody on eosinophils, airway hyper-responsiveness, and the late asthmatic response. Lancet 2000;356(9248):2144-2148. doi:https://doi.org/ 10.1016/S0140-6736(00)03496-6.

7. Flood-Page P, Swenson C, Faiferman I, Matthews J, Williams M, Brannick L, et al. A study to evaluate safety and efficacy of mepolizumab in patients with moderate persistent asthma. Am J Respir Crit Care Med. 2007;176(11):1062-71. https://doi.org/10. 1164/rccm.200701-085OC.

8. Pavord ID, Korn S, Howarth P, Bleecker ER, Buhl R, Keene ON, et al. Mepolizumab for severe eosinophilic asthma (DREAM): a multicentre, double-blind, placebo-controlled trial. Lancet. 2012;380(9842):651-9. https://doi.org/10.1016/s0140-6736(12) 60988-x.

9. Ortega HG, Liu MC, Pavord ID, Brusselle GG, FitzGerald JM, Chetta A, et al. Mepolizumab treatment in patients with severe eosinophilic asthma. N Engl J Med. 2014;371(13):1198-207. https://doi.org/10.1056/NEJMoa1403290.

10. Vannuvel K, Renard P, Raes M, Arnould T. Functional and morphological impact of ER stress on mitochondria. J Cell Physiol. 2013;228(9):1802-18. https://doi.org/10.1002/jcp.24360. 
11. Grootjans J, Kaser A, Kaufman RJ, Blumberg RS. The unfolded protein response in immunity and inflammation. Nat Rev Immunol. 2016;16(8):469-84. https://doi.org/10.1038/nri.2016.62.

12. Arensdorf AM, Diedrichs D, Rutkowski DT. Regulation of the transcriptome by ER stress: non-canonical mechanisms and physiological consequences. Front Genet. 2013;4:256. https://doi.org/10. 3389/fgene.2013.00256.

13. Wang M, Kaufman RJ. Protein misfolding in the endoplasmic reticulum as a conduit to human disease. Nature. 2016;529(7586): 326-35. https://doi.org/10.1038/nature17041.

14.• Kim SR, Lee YC. Endoplasmic reticulum stress and the related signaling networks in severe asthma. Allergy Asthma Immunol Res. 2015;7(2):106-17. https://doi.org/10.4168/aair.2015.7.2.106. Reviews how ER stress and UPR activation are closely interconnected to various signaling networks in cells, thereby contributing to the development of various immune and inflammatory disorders including corticosteroid-resistant severe allergic lung inflammation.

15. Lambrecht BN, Hammad H. The immunology of asthma. Nat Immunol. 2015;16(1):45-56. https://doi.org/10.1038/ni.3049.

16. Wenzel S, Ford L, Pearlman D, Spector S, Sher L, Skobieranda F, et al. Dupilumab in persistent asthma with elevated eosinophil levels. N Engl J Med. 2013;368(26):2455-66. https://doi.org/10. 1056/NEJMoa1304048.

17. Bleecker ER, FitzGerald JM, Chanez P, Papi A, Weinstein SF, Barker P, et al. Efficacy and safety of benralizumab for patients with severe asthma uncontrolled with high-dosage inhaled corticosteroids and long-acting beta2-agonists (SIROCCO): a randomised, multicentre, placebo-controlled phase 3 trial. Lancet. 2016;388(10056):2115-27. https://doi.org/10.1016/s01406736(16)31324-1.

18. Casale TB. Biologics and biomarkers for asthma, urticaria, and nasal polyposis. J Allergy Clin Immunol. 2017;139(5):1411-21. https://doi.org/10.1016/j.jaci.2017.03.006.

19. Corren J, Parnes JR, Wang L, Mo M, Roseti SL, Griffiths JM, et al. Tezepelumab in adults with uncontrolled asthma. N Engl J Med. 2017;377(10):936-46. https://doi.org/10.1056/NEJMoa1704064.

20. Wu W, Bleecker E, Moore W, Busse WW, Castro M, Chung KF, et al. Unsupervised phenotyping of severe asthma research program participants using expanded lung data. J Allergy Clin Immunol. 2014;133(5):1280-8. https://doi.org/10.1016/j.jaci.2013.11.042.

21. Ray A, Kolls JK. Neutrophilic inflammation in asthma and association with disease severity. Trends Immunol. 2017; https://doi.org/ 10.1016/j.it.2017.07.003. Summarizes evidence supporting the role of neutrophils in asthma severity and lung function. In addition, this article also focuses on the heterogeneity among neutrophil populations regarding the state of maturity/ activation in different patients with differences in the underlying pathophysiologies.

22. Wenzel SE. Asthma phenotypes: the evolution from clinical to molecular approaches. Nat Med. 2012;18(5):716-25. https://doi.org/ 10.1038/nm.2678.

23. Hastie AT, Moore WC, Meyers DA, Vestal PL, Li H, Peters SP, et al. Analyses of asthma severity phenotypes and inflammatory proteins in subjects stratified by sputum granulocytes. J Allergy Clin Immunol. 2010;125(5):1028-36.e13. https://doi.org/10.1016/ j.jaci.2010.02.008.

24. Woodruff PG, Modrek B, Choy DF, Jia G, Abbas AR, Ellwanger A, et al. T-helper type 2-driven inflammation defines major subphenotypes of asthma. Am J Respir Crit Care Med. 2009;180(5):388-95. https://doi.org/10.1164/rccm.2009030392OC.

25. Wenzel SE, Schwartz LB, Langmack EL, Halliday JL, Trudeau JB, Gibbs RL, et al. Evidence that severe asthma can be divided pathologically into two inflammatory subtypes with distinct physiologic and clinical characteristics. Am J Respir Crit Care Med.
1999;160(3):1001-8. https://doi.org/10.1164/ajrccm.160.3. 9812110.

26. Corren J, Busse W, Meltzer EO, Mansfield L, Bensch G, Fahrenholz J, et al. A randomized, controlled, phase 2 study of AMG 317, an IL-4Ralpha antagonist, in patients with asthma. Am J Respir Crit Care Med. 2010;181(8):788-96. https://doi.org/ 10.1164/rcem.200909-1448OC.

27. Kato T, Takeda Y, Nakada T, Sendo F. Inhibition by dexamethasone of human neutrophil apoptosis in vitro. Nat Immun. 1995;14(4): 198-208.

28. Moore WC, Meyers DA, Wenzel SE, Teague WG, Li H, Li X, et al. Identification of asthma phenotypes using cluster analysis in the severe asthma research program. Am J Respir Crit Care Med. 2010;181(4):315-23. https://doi.org/10.1164/rccm.2009060896OC. Using cluster analysis of $\mathbf{7 2 6}$ patients from the Severe Asthma Research Program (SARP), this article suggests five distinct clinical phenotypes of asthma seemingly having different pathophysiologic mechanisms. The results of study support the heterogeneity of severe asthma and the need for new approaches for the classification of disease severity in asthmatics.

29. McKinley L, Alcorn JF, Peterson A, Dupont RB, Kapadia S, Logar A, et al. TH17 cells mediate steroid-resistant airway inflammation and airway hyperresponsiveness in mice. J Immunol. 2008;181(6): 4089-97. https://doi.org/10.4049/jimmunol.181.6.4089.

30. Bullens DM, Truyen E, Coteur L, Dilissen E, Hellings PW, Dupont LJ, et al. IL-17 mRNA in sputum of asthmatic patients: linking T cell driven inflammation and granulocytic influx? Respir Res. 2006;7:135. https://doi.org/10.1186/1465-9921-7-135.

31. Busse WW, Holgate S, Kerwin E, Chon Y, Feng J, Lin J, et al. Randomized, double-blind, placebo-controlled study of brodalumab, a human anti-IL-17 receptor monoclonal antibody, in moderate to severe asthma. Am J Respir Crit Care Med. 2013;188(11):1294-302. https://doi.org/10.1164/rccm.2012122318OC.

32. Ray A, Raundhal M, Oriss TB, Ray P, Wenzel SE. Current concepts of severe asthma. J Clin Invest. 2016;126(7):2394-403. https://doi. org/10.1172/jci84144.

33. Kuo CS, Pavlidis S, Loza M, Baribaud F, Rowe A, Pandis I, et al. Thelper cell type 2 (Th2) and non-Th2 molecular phenotypes of asthma using sputum transcriptomics in U-BIOPRED. Eur Respir J. 2017;49(2) https://doi.org/10.1183/13993003.02135-2016. Through analyzing sputum cell transcriptomics from 104 moderate-to-severe asthmatic subjects and 16 non-asthmatic subjects from the U-BIOPRED cohort, one Th2 and two nonTh2 phenotypes of transcriptomic-associated clusters in gene expression were defined. Possible contribution of inflammasome-associated and metabolic/mitochondrial pathways in asthma severity were demonstrated in this group of patients.

34. Krug N, Madden J, Redington AE, Lackie P, Djukanovic R, Schauer U, et al. T-cell cytokine profile evaluated at the single cell level in BAL and blood in allergic asthma. Am J Respir Cell Mol Biol. 1996;14(4):319-26. https://doi.org/10.1165/ajrcmb.14.4. 8600935.

35. Corrigan CJ, Kay AB. CD4 T-lymphocyte activation in acute severe asthma. Relationship to disease severity and atopic status. Am Rev Respir Dis. 1990;141(4 Pt 1):970-7. https://doi.org/10.1164/ ajrccm/141.4_Pt 1.

36. Yu M, Eckart MR, Morgan AA, Mukai K, Butte AJ, Tsai M, et al. Identification of an IFN-gamma/mast cell axis in a mouse model of chronic asthma. J Clin Invest. 2011;121(8):3133-43. https://doi. org/10.1172/jci43598.

37. Randolph DA, Stephens R, Carruthers CJ, Chaplin DD. Cooperation between Th1 and Th2 cells in a murine model of 
eosinophilic airway inflammation. J Clin Invest. 1999;104(8): 1021-9. https://doi.org/10.1172/jci7631.

38. Ford JG, Rennick D, Donaldson DD, Venkayya R, McArthur C, Hansell E, et al. Il-13 and IFN-gamma: interactions in lung inflammation. J Immunol. 2001;167(3):1769-77. https://doi.org/10.4049/ jimmunol.167.3.1769.

39. Hetz C. The unfolded protein response: controlling cell fate decisions under ER stress and beyond. Nat Rev Mol Cell Biol. 2012;13(2):89-102. https://doi.org/10.1038/nrm3270.

40. Hollien J, Weissman JS. Decay of endoplasmic reticulum-localized mRNAs during the unfolded protein response. Science. 2006;313(5783):104-7. https://doi.org/10.1126/science.1129631.

41. Kim HJ, Jeong JS, Kim SR, Park SY, Chae HJ, Lee YC. Inhibition of endoplasmic reticulum stress alleviates lipopolysaccharideinduced lung inflammation through modulation of NF-kappaB/ HIF-1alpha signaling pathway. Sci Rep. 2013;3:1142. https://doi. org/10.1038/srep01142. Using a LPS-induced acute lung injury murine model, effects of ER stress inhibition on lung inflammation and transcriptional regulation of NF-KB/ HIF- $1 \alpha$ were examined. The results highlight the critical involvement of ER stress in acute neutrophilic lung inflammation.

42. Yoshida H, Matsui T, Yamamoto A, Okada T, Mori K. XBP1 mRNA is induced by ATF6 and spliced by IRE1 in response to ER stress to produce a highly active transcription factor. Cell. 2001;107(7):881-91. https://doi.org/10.1016/S0092-8674(01) 00611-0.

43. Marciniak SJ. Endoplasmic reticulum stress in lung disease. Eur Respir Rev. 2017;26(144). doi:https://doi.org/10.1183/16000617. 0018-2017.

44. Lambrecht BN, Hammad H. The airway epithelium in asthma. Nat Med. 2012;18(5):684-92. https://doi.org/10.1038/nm.2737.

45. Ribeiro CM, Boucher RC. Role of endoplasmic reticulum stress in cystic fibrosis-related airway inflammatory responses. Proc Am Thorac Soc. 2010;7(6):387-94. https://doi.org/10.1513/pats. 201001-017AW.

46. Martino ME, Olsen JC, Fulcher NB, Wolfgang MC, O'Neal WK, Ribeiro CM. Airway epithelial inflammation-induced endoplasmic reticulum $\mathrm{Ca} 2+$ store expansion is mediated by $\mathrm{X}$-box binding protein-1. J Biol Chem. 2009;284(22):14904-13. https://doi.org/10. 1074/jbc.M809180200.

47. Martino MB, Jones L, Brighton B, Ehre C, Abdulah L, Davis CW, et al. The ER stress transducer IRE1beta is required for airway epithelial mucin production. Mucosal Immunol. 2013;6(3):63954. https://doi.org/10.1038/mi.2012.105.

48. Kaser A, Lee AH, Franke A, Glickman JN, Zeissig S, Tilg H, et al. XBP1 links ER stress to intestinal inflammation and confers genetic risk for human inflammatory bowel disease. Cell. 2008;134(5): 743-56. https://doi.org/10.1016/j.cell.2008.07.021.

49. Moffatt MF, Kabesch M, Liang L, Dixon AL, Strachan D, Heath S, et al. Genetic variants regulating ORMDL 3 expression contribute to the risk of childhood asthma. Nature. 2007;448(7152):470-3. https://doi.org/10.1038/nature06014.

50. Bouzigon E, Corda E, Aschard H, Dizier MH, Boland A, Bousquet $\mathrm{J}$, et al. Effect of 17q21 variants and smoking exposure in earlyonset asthma. N Engl J Med. 2008;359(19):1985-94. https://doi. org/10.1056/NEJMoa0806604.

51.• Loser S, Gregory LG, Zhang Y, Schaefer K, Walker SA, Buckley J, et al. Pulmonary ORMDL3 is critical for induction of Alternariainduced allergic airways disease. J Allergy Clin Immunol. 2017;139(5):1496-507.e3. https://doi.org/10.1016/j.jaci.2016.07. 033. This study demonstrates the role of ORMDL3, a wellknown asthma susceptibility gene identified by genome-wide association studies, in the pathogenesis of Alternaria-induced allergic airway disease using ormdl3 knockout mice. These mice reveal that airway epithelial ORMDL3 plays a key role in the generation of fungal allergic airway disease via modulation of cellular stress pathways involving ATF6 arm of UPR.

52. Iwakoshi NN, Pypaert M, Glimcher LH. The transcription factor XBP-1 is essential for the development and survival of dendritic cells. J Exp Med. 2007;204(10):2267-75. https://doi.org/10.1084/ jem. 20070525.

53. Osorio F, Tavernier SJ, Hoffmann E, Saeys Y, Martens L, Vetters J, et al. The unfolded-protein-response sensor IRE-1alpha regulates the function of CD8alpha+ dendritic cells. Nat Immunol. 2014;15(3):248-57. https://doi.org/10.1038/ni.2808.

54. Shaffer AL, Shapiro-Shelef M, Iwakoshi NN, Lee AH, Qian SB, Zhao H, et al. XBP1, downstream of Blimp-1, expands the secretory apparatus and other organelles, and increases protein synthesis in plasma cell differentiation. Immunity. 2004;21(1):81-93. https:// doi.org/10.1016/j.immuni.2004.06.010.

55. Brunsing R, Omori SA, Weber F, Bicknell A, Friend L, Rickert R, et al. B- and T-cell development both involve activity of the unfolded protein response pathway. J Biol Chem. 2008;283(26):17954 61. https://doi.org/10.1074/jbc.M801395200.

56. Kamimura D, Bevan MJ. Endoplasmic reticulum stress regulator XBP-1 contributes to effector CD8+ T cell differentiation during acute infection. J Immunol. 2008;181(8):5433-41. https://doi.org/ 10.4049/jimmunol.181.8.5433.

57. Iwakoshi NN, Lee AH, Vallabhajosyula P, Otipoby KL, Rajewsky $\mathrm{K}$, Glimcher LH. Plasma cell differentiation and the unfolded protein response intersect at the transcription factor XBP-1. Nat Immunol. 2003;4(4):321-9. https://doi.org/10.1038/ni907.

58. Osborne LC, Monticelli LA, Nice TJ, Sutherland TE, Siracusa MC, Hepworth MR, et al. Coinfection. Virus-helminth coinfection reveals a microbiota-independent mechanism of immunomodulation. Science. 2014;345(6196):578-82. https://doi.org/10.1126/science. 1256942.

59. Sutherland TE, Logan N, Ruckerl D, Humbles AA, Allan SM, Papayannopoulos V, et al. Chitinase-like proteins promote IL-17mediated neutrophilia in a tradeoff between nematode killing and host damage. Nat Immunol. 2014;15(12):1116-25. https://doi.org/ 10.1038/ni.3023.

60.• Bettigole SE, Lis R, Adoro S, Lee AH, Spencer LA, Weller PF, et al. The transcription factor XBP1 is selectively required for eosinophil differentiation. Nat Immunol. 2015;16(8):829-37. https:// doi.org/10.1038/ni.3225. Through using multiple genetic and cellular models, this study identifies an unexpectedly selective and absolute requirement for the UPR-associated transcription factor, XBP1, in eosinophil development without an effect on the survival of basophils or neutrophils. This finding implies the importance of secretory-pathway homeostasis in the pathogenesis of type 2 allergic immune response.

61. Martinon F, Chen X, Lee AH, Glimcher LH. TLR activation of the transcription factor XBP1 regulates innate immune responses in macrophages. Nat Immunol. 2010;11(5):411-8. https://doi.org/10. 1038/ni.1857.

62. Woo CW, Cui D, Arellano J, Dorweiler B, Harding H, Fitzgerald $\mathrm{KA}$, et al. Adaptive suppression of the ATF4-CHOP branch of the unfolded protein response by toll-like receptor signalling. Nat Cell Biol. 2009;11(12):1473-80. https://doi.org/10.1038/ncb1996.

63.• Kim SR, Kim DI, Kang MR, Lee KS, Park SY, Jeong JS, et al. Endoplasmic reticulum stress influences bronchial asthma pathogenesis by modulating nuclear factor kappaB activation. J Allergy Clin Immunol. 2013;132(6):1397-408. doi:https://doi.org/10.1016/ j.jaci.2013.08.041. Using mice sensitized with ovalbumin and LPS and challenged with ovalbumin, a neutrophilic non-type 2 asthma murine model, this study demonstrates a novel concept of bronchial asthma pathogenesis involving ER stress, for the first time. Crucial role of ER stress in asthma is further highlighted by the data that ER stress markers in PBMCs and bronchoalveolar lavage fluids from human asthmatic 
patients are remarkably increased compared to those from healthy subjects.

64. Hoffman SM, Tully JE, Nolin JD, Lahue KG, Goldman DH, Daphtary N, et al. Endoplasmic reticulum stress mediates house dust mite-induced airway epithelial apoptosis and fibrosis. Respir Res. 2013;14:141. https://doi.org/10.1186/1465-9921-14-141.

65. Hoffman SM, Chapman DG, Lahue KG, Cahoon JM, Rattu GK, Daphtary N, et al. Protein disulfide isomerase-endoplasmic reticulum resident protein 57 regulates allergen-induced airways inflammation, fibrosis, and hyperresponsiveness. J Allergy Clin Immunol. 2016;137(3):822-32.e7. https://doi.org/10.1016/j.jaci.2015.08. 018.

66. Makhija L, Krishnan V, Rehman R, Chakraborty S, Maity S, Mabalirajan U, et al. Chemical chaperones mitigate experimental asthma by attenuating endoplasmic reticulum stress. Am J Respir Cell Mol Biol. 2014;50(5):923-31. https://doi.org/10.1165/rcmb. 2013-0320OC

67. Zhang K, Kaufman RJ. From endoplasmic-reticulum stress to the inflammatory response. Nature. 2008;454(7203):455-62. https:// doi.org/10.1038/nature07203.

68.• Lee KS, Jeong JS, Kim SR, Cho SH, Kolliputi N, Ko YH, et al. Phosphoinositide 3-kinase-delta regulates fungus-induced allergic lung inflammation through endoplasmic reticulum stress. Thorax. 2016;71(1):52-63. https://doi.org/10.1136/thoraxjnl-2015-207096. Using Aspergillus fumigatus-induced in vivo and in vitro experimental systems, critical roles of phosphoinositide 3kinase delta signaling in the regulation of ER stress/ mitochondrial oxidative stress in the pathogensis of corticosteroid-resistant eosinophilic fungal asthma are demonstrated. A representative ER stress marker, GRP78, is also increased in lung tissues of patients with ABPA, which is a complex hypersensitivity reaction often occurs in patients with asthma or cystic fibrosis when bronchi become colonized by Aspergillus species.

69. Denning DW, O'Driscoll BR, Hogaboam CM, Bowyer P, Niven RM. The link between fungi and severe asthma: a summary of the evidence. Eur Respir J. 2006;27(3):615-26. https://doi.org/10. $1183 / 09031936.06 .00074705$. The pivotal article greatly reviews the epidemiological evidence of fungal implication in the severity of asthma and discusses possible pathogenic mechanisms. Furthermore, authors for the first time propose a new label for patients who have severe asthma and evidence of fungal sensitization, namely SAFS.

70. Zureik M, Neukirch C, Leynaert B, Liard R, Bousquet J, Neukirch F. Sensitisation to airborne moulds and severity of asthma: cross sectional study from European Community respiratory health survey. BMJ. 2002;325(7361):411-4. https://doi.org/10.1136/bmj. 325.7361.411.

71. Denning DW, Pashley C, Hartl D, Wardlaw A, Godet C, Del Giacco $\mathrm{S}$, et al. Fungal allergy in asthma-state of the art and research needs. Clin Transl Allergy 2014;4:14. doi:https://doi.org/10.1186/20457022-4-14.

72. O'Driscoll BR, Powell G, Chew F, Niven RM, Miles JF, Vyas A, et al. Comparison of skin prick tests with specific serum immunoglobulin $\mathrm{E}$ in the diagnosis of fungal sensitization in patients with severe asthma. Clin Exp Allergy. 2009;39(11):1677-83. https://doi. org/10.1111/j.1365-2222.2009.03339.x.

73. Bronner DN, Abuaita BH, Chen X, Fitzgerald KA, Nunez G, He Y, et al. Endoplasmic reticulum stress activates the inflammasome via NLRP3- and caspase-2-driven mitochondrial damage. Immunity. 2015;43(3):451-62. https://doi.org/10.1016/j.immuni.2015.08.008.

74. Kim SR, Kim HJ, Kim DI, Lee KB, Park HJ, Jeong JS, et al. Blockade of interplay between IL-17A and endoplasmic reticulum stress attenuates LPS-induced lung injury. Theranostics. 2015;5(12):1343-62. https://doi.org/10.7150/thno.11685. Using both in vivo and in vitro experimental systems for LPSinduced acute neutrophilic lung inflammation, authors demonstrate that LPS-induced ER stress leads to the increased expression of IL-17 in airway epithelium, thereby further potentiating ER stress and NF- $\mathrm{KB}$ activation via forming a positive feedback loop in airway epithelial cells. 